\title{
Localization of candidate regions for a novel gene for Kartagener syndrome
}

\author{
Ilse Gutierrez-Roelens ${ }^{1}$, Thierry Sluysmans ${ }^{2}$, Mark Jorissen $^{3}$, Mustapha Amyere ${ }^{1}$ and \\ Miikka Vikkula ${ }^{1 *}$
}

${ }^{1}$ Laboratory of Human Molecular Genetics, Christian de Duve Institute of Cellular Pathology, Brussels, Belgium;
${ }^{2}$ Division of Pediatric Cardiology, Cliniques universitaires St Luc, Université catholique de Louvain, Brussels, Belgium
and ${ }^{3}$ Department of Medical Genetics, Universitaire Ziekenhuizen of Leuven, Leuven, Belgium

Asymmetric positioning of internal organs is a characteristics of vertebrates. The normal left-right anatomic positioning, situs solitus, sometimes does not occur normaly, leading to laterality defects. Studies in animal models have shown that laterality decisions are mediated by a cascade of genes that lead to the asymmetric expression of Nodal, LEFTA, LEFTB and PITX2 in the lateral plate mesoderm. A search for mutations in genes implicated in left-right patterning in animal models allowed genes associated with heterotaxia defects in humans to be identified. However, these genes explain only a small percentage of human situs defects, suggesting that other genes must play a role. In this study, we report a consanguineous family of Turkish origin, composed of two unaffected parents and three children, two of whom presented Kartagener syndrome. On the basis of their family history, we hypothesize autosomal recessive mode of inheritance. A genotype analysis with polymorphic markers did not show linkage with any known genes or loci causing laterality disorders. Array CGH did not detect a duplication or microdeletion greater than $1 \mathrm{Mb}$ as a possible cause. Genome wide screening using $10 \mathrm{~K}$ Affymetrix SNP chips was performed, allowing the identification of two regions of autozygosity, one in chromosome 1 and the other on chromosome 7 . In the chromosome 1 locus, a strong candidate gene, encoding the kinesinassociated protein 3 (KIF3AP) was not mutated, based on SSCP/heteroduplex analysis and direct sequencing. These data provide a basis for the identification of a novel gene implicated in Kartagener syndrome.

European Journal of Human Genetics (2006) 14, 809-815. doi:10.1038/sj.ejhg.5201631; published online 26 April 2006

Keywords: heart; Kartagener syndrome; left-right patterning; locus

\section{Introduction}

Internal organs display highly conserved asymmetry in vertebrates regarding their placement and the looping of the heart. Failure to correctly establish left-right (L/R) patterning results in situs inversus, a complete mirror image

*Correspondence: Dr Miikka Vikkula, Laboratory of Human Molecular Genetics, Christian de Duve Institute of Cellular Pathology and Université catholique de Louvain, Avenue Hippocrate $74+5$, bp 75.39. Brussels, B-1200, Belgium. Tel: + 32276474 90; Fax: + 3227647460 ; E-mail: vikkula@bchm.ucl.ac.be

Received 12 May 2005; revised 6 February 2006; accepted 28 February 2006; published online 26 April 2006 reversal, isomerism, duplication of the left or the right side, or situs ambiguous, an intermediate phenotype. Despite our limited knowledge of the pathogenesis of laterality defects, molecular studies in chicken, frog, zebrafish and mouse have given insights into genes implicated in leftright patterning.

In man, left-right defects exhibit locus heterogeneity, and autosomal dominant, autosomal recessive and $\mathrm{X}$ linked inheritance patterns have been described. A search for mutations in genes causing laterality defects in animal models implicated ACVR2B, CRYPTIC, LEFTYA, and $I N V .^{1-4}$ Linkage analysis resulted in the identification of 


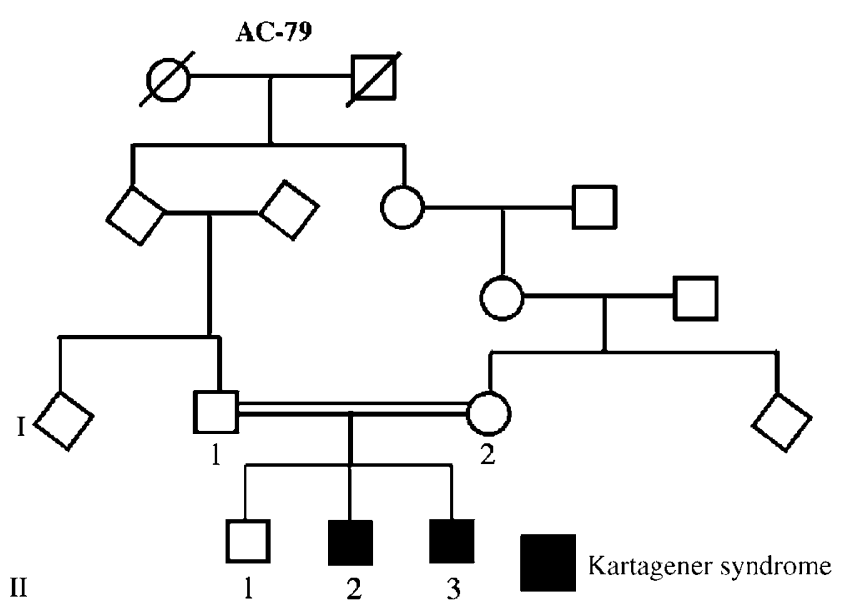

Figure 1 Pedigree of family AC-79.

the ZIC3 gene in some laterality defects, ${ }^{5}$ and mutations in the gene encoding connexin 43 were reported in patients with visceroatrial heterotaxy syndrome. ${ }^{6}$ However, these results were not confirmed. In addition, cytogenetic rearrangement led to the localization of PA26, a candidate gene which is disrupted in a patient with abdominal situs inversus, ${ }^{7}$ and mutations in CRELD1 and CSX/NKX2-5 have been associated with heterotaxy in two cases ${ }^{8,9}$ Finally, a link between cilia and L/R determination has been suspected in man, since primary ciliary dyskinesia (PCD), an autosomal recessive disorder, is characterized by loss of motility of respiratory cilia and sperm flagella. In $50 \%$ of the PCD patients, situs inversus is also present leading to Kartagener syndrome. To date, four dynein genes (DNAI1, DNAH5, DNAH7 and DNAH11) have been identified to be mutated in PCD and Kartagener syndrome ${ }^{10-13}$

Here, we report a consanguineous family composed of healthy parents and three children, two of whom present Kartagener syndrome (Figure 1). All known genes were excluded as the cause of the laterality defect by linkage analysis, and no deletions or amplifications greater than $1 \mathrm{Mb}$ were identified by array CGH. Genome wide SNP and STRP screening for homozygosity allowed us to identify novel linked regions, providing the basis for the identification of a new gene for Kartagener syndrome.

\section{Materials and methods}

\section{Clinical data of family AC-79}

Family AC-79 was clinically examined by Th.S. Written informed consent was obtained from the two adult participants for their own and their children's participation, in accordance with the ethics committee of Faculty of Medicine, Université catholique de Louvain. Blood samples were collected from the five participants. Genomic DNA was extracted from the buffy coat (Qiagen DNA extraction Kit, The Netherlands).

Family AC-79 is composed of five individuals. The parents and one of the children (II-1) are unaffected, whereas the two other children (II-2 and II-3) are affected. The parents are consanguineous, as shown in the pedigree (Figure 1). Individual II-2 has dextrocardia with concordant atrioventricular and ventriculoarterial connections (inverted normal heart $\{\mathrm{I}, \mathrm{L}, \mathrm{I}\}$, according to Van Praagh $\mathrm{R}$ segmental approach to diagnosis). He has no cardiac defect. Individual II-3 has a complex congenital heart disease with dextrocardia, situs inversus of the atria, persistence of the right and left superior vena cava with absent innominate vein, an unroof coronary sinus, an atrial septal defect, discordant atrioventricular connection with congenitally corrected transposition of the great arteries (or $\{\mathrm{I}, \mathrm{D}, \mathrm{D}\}$, physiologically corrected transposition of the great arteries, according to Van Praagh R segmental approach to diagnosis), pulmonary atresia, and a large ventricular septal defect. He also has decreased mucociliary transport and chronic respiratory disease. He had a right and a left Blalock-Taussig shunt performed.

Nasal biopsies were taken for individuals II-2 and II-3 in order to diagnose Kartagener syndrome. Ciliary ultrastructure was normal in both biopsies. Ciliary ultrastructure and beat frequency were also analysed after cell culture. For individual II-2, the outer and inner dynein arms were absent after ciliogenesis and the cilia were immotile or did not present a coordinated movement. The same result was observed in a second nasal biopsy. For individual II-3, the ciliary culture did not show any ultrastructural abnormality. However, the cilia were immotile. Based on the clinical features, and the ciliary functional and ultrastructural analysis for individuals II-2 and II-3, both were considered to be affected with the Kartagener syndrome.

Examination of individual II-1 did not show any clinical manifestation of primary ciliary dyskinesia or Kartagener syndrome. No ciliary ultrastructural or functional analysis was performed. He was considered to be unaffected.

\section{Identification of candidate genes and genome wide screening}

Genes and loci responsible for abnormal left-right patterning were screened with short tandem repeat markers. The STRPs were selected on the basis of their map position and heterozygozity (minimal heterozygosity of 0.7 ) from the human genome assembly hg12 (NCBI build 30) till the latest version database hg17 (NCBI build 35) from the UCSC Genome Browser and Blat server (http://www.genome. ucsc.edu/). For each marker, we designed and synthesized new primer pairs (synthesized by Invitrogen-Life Technologies or Eurogentec, Belgium). This allowed us to obtain clearer results for these polymorphisms (primers available on request). Genotyping of individuals, using ${ }^{32} \mathrm{P}$-labeled oligonucleotides was performed, as described elsewhere. 
Genome wide screening was performed with polymorphic markers from the Weber set 8.0 (Research Genetics). These markers are evenly distributed, allowing a genomic scan with a $10 \mathrm{cM}$ resolution. For each marker, the genotype of the five individuals of family AC-79 was determined so as to detect regions of autozygosity for the two affected children.

\section{Search for human paralogues or homologues}

To identify functional candidate genes, we searched for paralogues of proteins that are encoded by genes responsible for laterality disorders. The amino-acid sequences were analysed in BLAT server (UCSC). Novel candidate genes were selected on the basis of high percentage of identity and after the examination of the sequence alignment quality (Table 2). Homozygosity was tested as described above.

\section{Search for microdeletions using array CGH (aCGH)}

Whole genome aCGH from individual II-3 was performed with Spectral's Genomic (G) Chip ${ }^{\mathrm{TM}}$ Human BAC Arrays-1 MbV1.2 (Genosystems, Paris, France). The array contained 2500 nonoverlapping BAC clones spotted in duplicate. The BACs span the genome with an approximately $1 \mathrm{Mb}$ cloneto-clone interval. Genomic DNA $(1 \mu \mathrm{g})$ was digested with $40 \mathrm{U}$ of EcoR1. Labeling, hybridization and washing were performed according to the manufacturer's protocol (http://www.spectralgenomics.com). The array was scanned using a GenePix Axon 4000B scanner. The GenePix $^{\mathrm{TM}}$ Pro 4.1 software was used for image acquisition and image analysis. The scanned data was analysed using the SpectralWare ${ }^{\mathrm{TM}}$ BAC Array Analysis Software.

\section{High-resolution SNP-based genomic screening}

Genomic DNA from the five AC-79 members were hybridized on the Affymetrix GeneChip ${ }^{\mathrm{R}}$ Human Mapping $10 \mathrm{~K}$ Arrays according to the manufacturer's protocol (http://www.affymetrix.com). Each array has 11555 SNPs and the median physical distance between SNPs is approximately $105 \mathrm{~Kb}$. The arrays were scanned using the Affymetrix Gene Chip ${ }^{\mathrm{R}}$ Scanner 3000 with the Gene Chip Operating System 1.0 (GCOS) and the data was analysed using the Gene Chip DNA Analysis Software 2.0 (GDAS). The analysed data was classified by chromosome and sorted based on the physical position of the SNPs. We looked for regions with informative SNPs that were homozygous for the same allele for the two affected children and heterozygous for the parents, and heterozygous or homozygous for the opposite allele for the unaffected child. Autozygosity scores were calculated with the Genespring GT software (Silicon Genetics) using an algorithm based on that of Broman and Weber. ${ }^{14}$ The software calculates the likelihoods for genomic regions to be identical by descent. Two-point Lod scores are first calculated for each affected individual. This takes into account the allele frequencies in a known population and tests the null hypothesis of nonautozygosity against autozygosity. The regional score is the summation of twopoint Lod data across a region of autozygosity (details on the generation of regional scores can be found at http:// www.silicon genetics.com/support/autozygosity.pdf). The autozygous regions with the highest regional scores are the best candidate regions. This method was validated by Puffenberger et al. ${ }^{15}$ In our analysis, penetrance was set at $100 \%$ and phenocopy rate at $0 \%$.

\section{KIF3AP mutation screening}

To perform SSCP/heteroduplex screening and direct sequencing of the KIF3AP positional candidate gene, primer pairs were designed based on the published cDNA sequence (GenBank NM 014970) and on the UCSC Human Genome Browser (accession U5991). Twenty-seven primer pairs were synthesized (Eurogentec, Belgium) (primers sequences and PCR conditions are available on request) to amplify the 20 exons and the exon/intron boundaries. The sizes of the amplification products were $165-268 \mathrm{bp}$. SSCP and heteroduplex analysis were performed as previously described. ${ }^{16}$ Fragments showing abnormal migration were reamplified, purified (Qiaquick PCR Purification Kit, Qiagen, The Netherlands) and sequenced on Beckman CEQ 2000 capillary sequencer with the CEQ Dye Terminator Cycle Sequencing Quick Start Kit (Analis, Belgium). Sequences were analysed using the Sequencher software (version 4.1.2, Gene Codes Corp., Ann Arbor, MI, USA).

\section{Results \\ Candidate genes and loci screened}

We hypothesize an autosomal recessive mode of inheritance for the Kartagener syndrome in family AC-79 on the basis of consanguinity, both parents being unaffected, and two children being affected by the same rare disorder. Accordingly, we expect the affected children to be homozygous for the disease causing locus, the parents to be heterozygous for an informative marker, and the unaffected child to be heterozygous or homozygous for the other allele. We first screened genes previously identified to be causative of heterotaxy syndromes in man (Table 1). Six polymorphic markers, in a maximal interval of $8 \mathrm{Mb}$ spanning each gene, were genotyped for the five individuals. None of the markers were homozygous for the affected individuals II-2 and II-3, and heterozygous for the unaffected individuals I-1, I-2 and II-1. Eight new candidate genes were then selected on the basis of homology (paralogues or orthologues) to proteins implicated in laterality disorders (Table 2). Additional candidate loci were selected on the basis of reported cytogenetic defects observed in heterotaxia patients and linkage analysis (Table 2). Finally, murine models with left-right defects permitted us to identify an additional nine candidate genes 
Table 1 Tested loci (genes that are known to be mutated in patients with heterotaxy or Kartagener syndrome)

\begin{tabular}{|c|c|c|c|c|}
\hline Gene & Locus & PCD/Kartagener & OMIM & References \\
\hline CX43 & $6 q 21-q 23.2$ & & 121014 & Britz-Cunningham et al $1995^{6}$ \\
\hline $\mathrm{ZIC3}$ & $\mathrm{Xq} 26.3$ & & 300265 & Gebbia et al $1997^{5} /$ Megarbane et al $2000^{17} /$ Ware et al $2004^{18}$ \\
\hline ACVR2B & $3 p 22-p 21.3$ & & 602730 & Kosaki et al $1999^{1}$ \\
\hline LEFTYA & $1 \mathrm{q} 42.1$ & & 601877 & Kosaki et al $1999^{1}$ \\
\hline DNAI1 & $9 p 13.3$ & + & 604366 & Pennarun et al $1999^{19} /$ Guichard et al $2001^{10}$ \\
\hline CFC-1 & $2 q 21.1$ & & 605194 & Bamford et al $2000^{2} /$ Goldmuntz et al $2002^{20}$ \\
\hline DNAH5 & $5 p 14-p 15$ & + & 603335 & Olbrich et al $2002^{11}$ \\
\hline DNAH11 & $7 \mathrm{p} 21$ & + & 603339 & Bartoloni et al $2002^{13}$ \\
\hline DNAH7 & $2 q 32.3$ & + & - & Zhang et al $2002^{12}$ \\
\hline
\end{tabular}

Table 2 Candidate genes

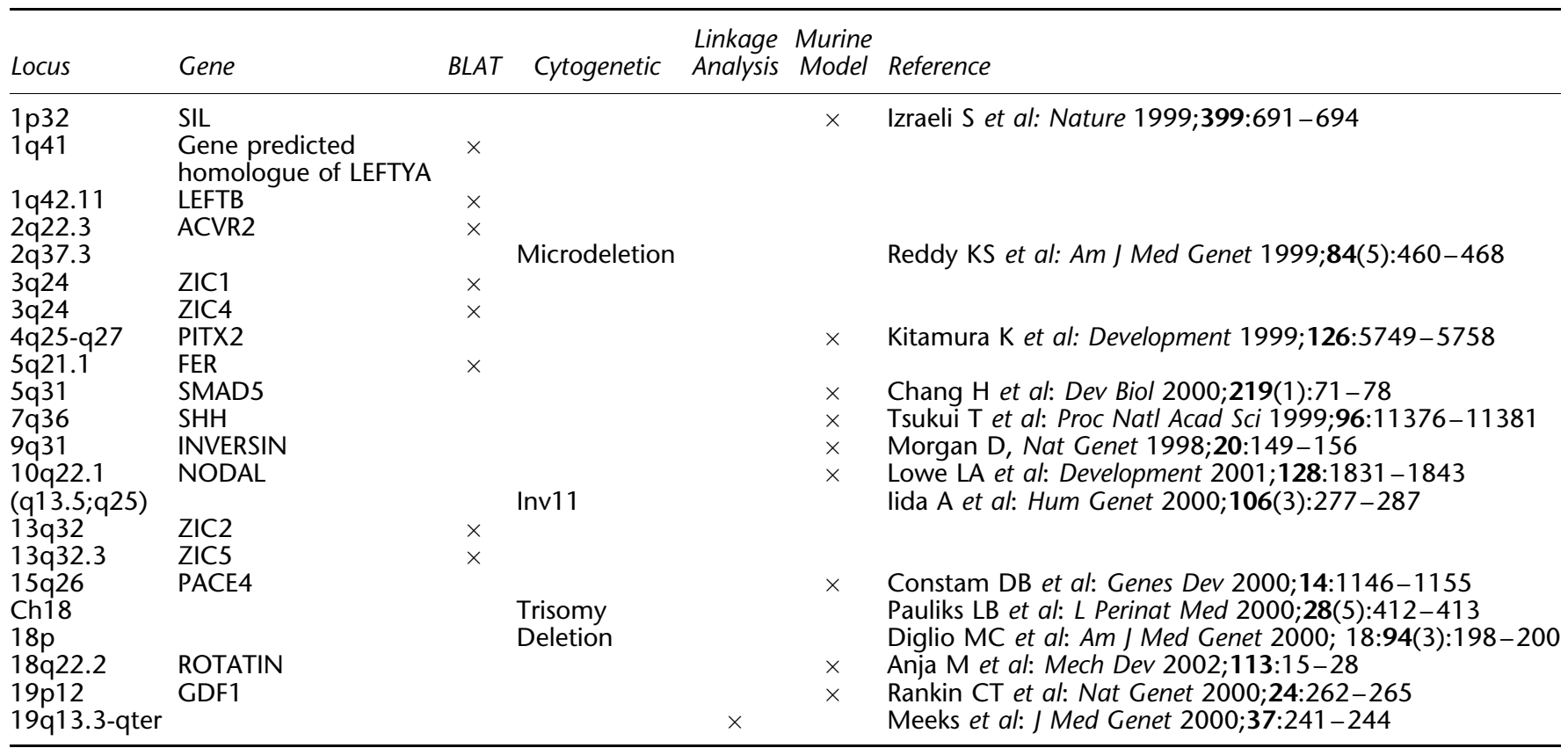

Candidate genes were selected on the basis of homology to Table 1 genes (paralogues) or to murine heterotaxy-associated genes (homologues). In addition, four candidate loci are based on reported cytogenetic abnormalities and one on linkage analysis.

(Table 2). Genotyping did not show proof for linkage to any of these loci $(n=22)$.

\section{Array CGH}

In order to examine the presence of a microdeletion (or amplification) as the cause of the Kartagener syndrome, we performed array CGH on genomic DNA from one affected individual (II-3). We identified amplification in one BAC clone (cloneRP11-79F15) on chromosome 19. However, this is a known polymorphism. The array CGH used has a density of $1 \mathrm{BAC} / \mathrm{Mb}$ and no deletion was observed.

\section{Genome wide screening for homozygosity}

Genome wide screening performed with polymorphic markers from the Weber set 8.0 , allowed us to identify 10 possible shared homozygous regions and 29 noninformative regions. Additional markers were genotyped in all the regions to define the critical interval and to exclude or to increase informativeness in the uninformative regions. Owing to the small size of the family, this led to a limited increase in information. Thus, we decided to refine the genome analysis using the Affymetrix GeneChip ${ }^{\mathrm{R}}$ Human Mapping $10 \mathrm{~K}$ Array. The homozygous regions that were found with STRP markers overlapped with those observed with SNPs. We identified 44 homozygous regions, from which 41 were between 0.1 and $6 \mathrm{Mb}$ in size. The expected linked region to the disease should be between 10 and $30 \mathrm{Mb}$ on the basis of the family structure. Thus, these 41 homozygous regions were considered unlikely to contain the mutated gene and three regions retained 
our attention. From these, two are highly informative, one in chromosome 1 (spread over $44.6 \mathrm{Mb}$ ), and a second in chromosome $7(13.7 \mathrm{Mb})$. The third region in chromosome $19(12 \mathrm{Mb})$ contains $8.7 \mathrm{Mb}$, which is not covered

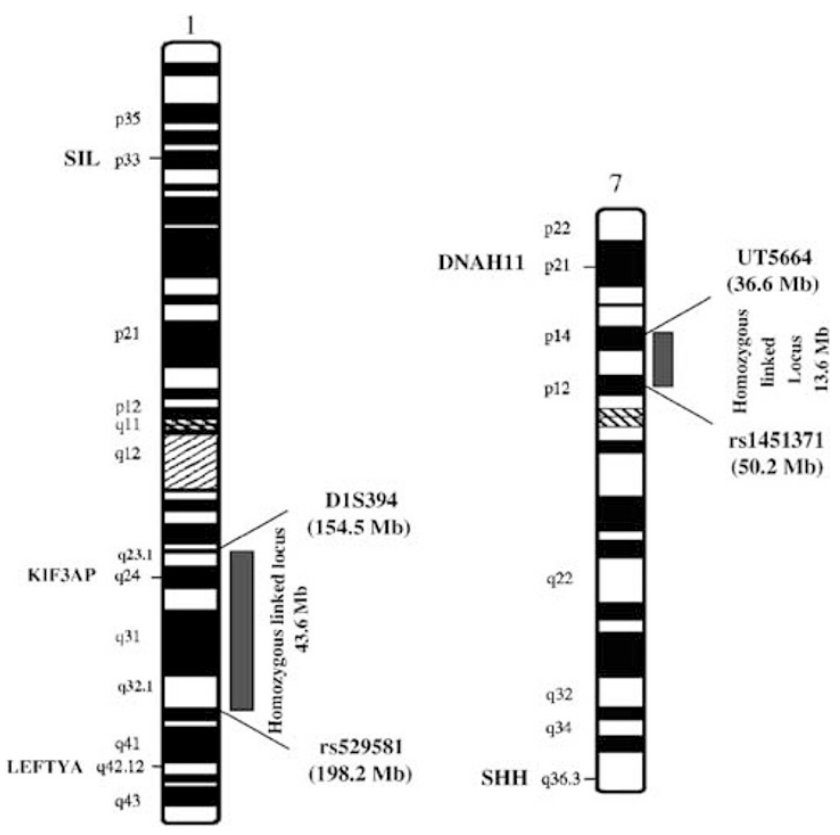

Figure 2 Candidate homozygous regions on chromosomes 1 and 7 for a novel gene for Kartagener syndrome. For each locus, the most telomeric and centromeric homozygous marker is shown. with SNPs on the Affymetrix Chip and thus is poorly informative.

To reduce the linked regions, we genotyped additional polymorphic microsatellites identified in databases. For the pericentromeric locus in chromosome 19, we genotyped five additional markers at both ends of the region. We reduced the interval to $10.4 \mathrm{Mb}$ with two markers that were heterozygous from each side of the gap. For chromosome 1, genotyping of an additional 21 markers in the $44.6 \mathrm{Mb}$ locus permitted us to cut $1 \mathrm{Mb}$. Finally, for chromosome 7 , the 10 additional markers genotyped to the extremities of the homozygous region did not give additional information.

Regional and two-point Lod scores were calculated for the family data with Genespring GT. When the analysis is based on a Caucasian control population, the highest regional Lod scores were identified for the candidate regions on chromosome 1 (74.31) and 7 (25.24). When using the recent HapMap data, the chromosome 1 locus remained the most strongly linked with a regional Lod score of 12.97, compared to that of 2.58 for the chromosome 7 locus (Figure 3).

Based on these results, we have one very strong candidate region in chromosome 1 between markers D1S394 and rs529581 (43.6 Mb) 1q23.1-q32.1, and a second candidate locus in chromosome 7 , between UT5664 and rs1451371 (13.6 Mb) 7p14.2-p12.2 (Figure 2). In addition, we could not exclude the gaps that were uncovered with Affymetrix SNPs. a

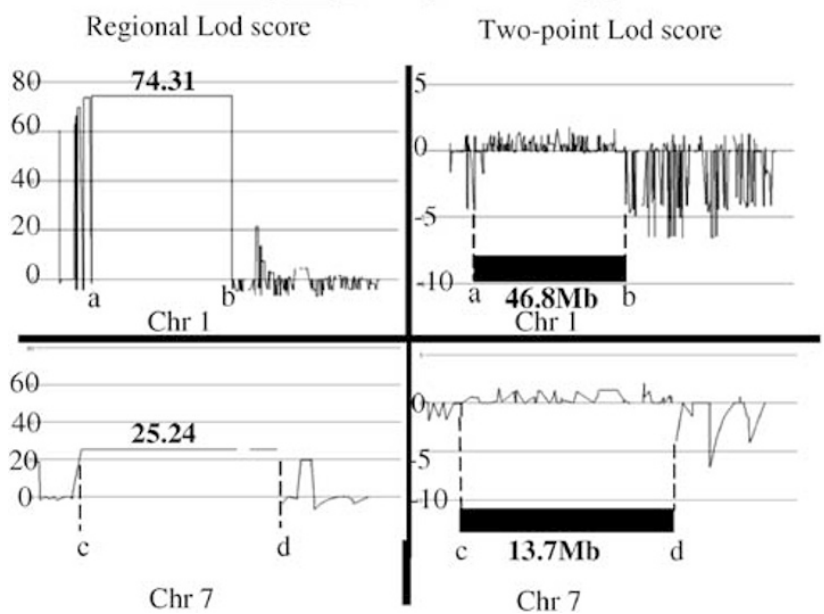

b

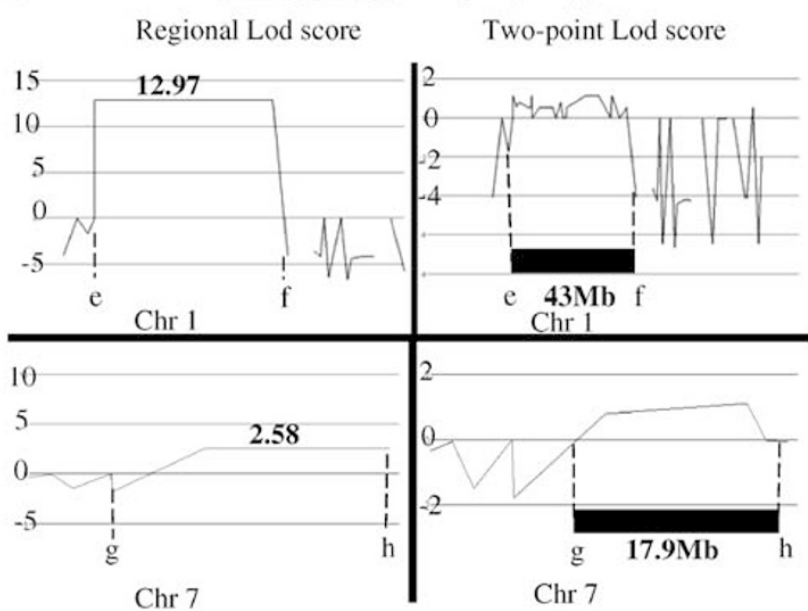

Figure 3 Regional and two-point Lod scores calculated by Genespring GT based on a Caucasian population (a) and the HapMap (b). The highest regional Lod scores of 74.31 and 25.24, when comparing the family data with a Caucasian population were found, in chromosome 1 and 7 , respectively. The regions are delimited by SNPs rs2335230 (a) at position $151402701 \mathrm{Mb}$ and rs614452 (b) at position $198285220 \mathrm{Mb}$ for chromosome 1 and SNPs rs2016547 (c) at position $36563547 \mathrm{Mb}$ and rs 1451371 (d) at position $50327260 \mathrm{Mb}$ for chromosome 7. When analysing the family using the HapMap data, the chromosome 1 locus stays as the most likely locus with a regional Lod score of 12.97, compared to that of 2.58 for the chromosome 7. For chromosome 1 the region was delimited by the two negative SNPs rs1342947 (e) at position $155272787 \mathrm{Mb}$ and rs 951908 (f) at position $19245141 \mathrm{Mb}$. For chromosome 7, the region was delimited by the SNPs rs 1419794 (g) at position $34474245 \mathrm{Mb}$ and rs 1822228 (h) at position $52434790 \mathrm{Mb}$. 
KIF3AP kinesin associated protein mutation screening SSCP screening of the KIF3AP gene allowed us to detect one abnormally migrating fragment for individuals I-1, I-2 and II-1. The fragment was sequenced and a c.-243 G>A nucleotide change in the $5^{\prime} \mathrm{UTR}$ of the gene was detected. The unaffected parents (I-1, I-2) and the child II-1 were heterozygous and the affected children (II-2, II-3) were homozygous for the A allele. This variation has been described as a frequent SNP in databases (rs1360294). Direct sequencing of the entire KIF3AP gene and exon/ intron boundaries, was also performed. No other nucleotide change was observed. As all the 20 exons and exon/ intron boundaries were amplified for the five individuals, deletions in these regions were excluded. However, we did not exclude duplications, inversions, or promoter mutations.

\section{Discussion}

In this paper, we report a consanguineous family of Turkish origin, composed of two affected children with Kartagener syndrome. All known loci and genes reported to be mutated in heterotaxy syndromes in man were excluded. We screened new candidate loci that contain gene homologues to known causative genes, or that have been observed to contain cytogenetic defects in heterotaxia patients, or that have been implied in animal models of left-right definition defects. None of these showed proof of linkage. Large microdeletions or amplifications were excluded by aCGH. In contrast, genome wide screening for homozygosity with STRPs and Affymetrix $10 \mathrm{~K}$ SNP chip allowed us to identify novel candidate loci for Kartagener syndrome, the strongest of which is the one on chromosome 1q23.1-q32.1. Another possible locus lies on chromosome 7p14.2-p12.2.

Altogether, we identified 44 regions of homozygosity. However, the majority were very small. By autozygosity calculations, the highest regional Lod scores were identified for the regions on chromosome 1 (74.31) and 7 (25.24), when the family data was compared with a Caucasian population. These two regions were defined by consecutive two-point Lod scores that were all positive (maximum two-point Lod score of 1.775 for chromosome 1 and 2.363 for chromosome 7). Centromeric and telomeric to these regions, consecutive two-point Lod scores were negative, excluding these regions and delimiting the autozygous loci. The regional Lod scores for the other 42 regions were below 10. Similar results were obtained when the data was compared with HapMap data. The pericentromeric region in chromosome 19 , which could not be excluded, gave a regional Lod score of -0.1360 . On the basis of Puffenberger et al, ${ }^{15}$ the region with the highest autozygosity score is the most likely locus for the defective gene. Based on this assumption and on the expected size of the linked region, we considered the 41 homozygous regions between 0.1 and $6 \mathrm{Mb}$ in size to be weak candidates and the two regions on chromosome 1 and 7, with the highest autozygosity scores and the largest sizes, as the best candidates.

It is important to keep in mind that the mode of inheritance in family AC-79 could also be recessive $\mathrm{X}$ linked. In this case, individual I-2 (mother of the children) must be a carrier of the $\mathrm{X}$ allele linked to the Kartagener syndrome. The two affected boys should have inherited this $\mathrm{X}$ allele and the unaffected child the opposite $\mathrm{X}$ allele. Based on this mode of inheritance, we could only partially exclude the $X$ chromosome with the Weber set 8.0 and with the Affymetrix SNP chip. Individual I-2 was not very heterozygous and thus two big uninformative regions remained, the first in Xp22.31-21.3 of $17 \mathrm{Mb}$ of size (between SNP rs1921706 and rs4131175) and the second in Xp11.3-q25 of $75.54 \mathrm{Mb}$ of size (between STRP marker GATA144D04 and rs2353720). Budny and co-workers reported a mutation in the OFD1 gene causing a new syndrome characterized by mental retardation, primary ciliary dyskinesia and macrocephaly, but without heterotaxy, in a three-generation family with X-linked recessive inheritance (Budny et al, ESHG meeting, Prague May 2005, 13-supplement 1,65-66). The OFD1 gene is located in the

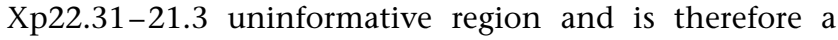
possible candidate gene.

In the best candidate regions of chromosome 1 and 7, we also identified possible candidate genes for left-right patterning. The kinesin associated protein 3 (KIF3AP) gene is located in chromosome 1q23.1-1q32.1. This protein has been predicted to mediate cargo binding by kinesin II (KIF3A/3B), although its precise function remains unknown. ${ }^{21}$ However, kinesin superfamily proteins (KIFs) are motor proteins that play an important role in intracellular trafficking, such as in intraciliary-flagellar transport. In addition, Kif $3 A^{-/-}$and $k i f 3 B^{-/-}$mice did not survive beyond mid-gestation, and displayed severe developmental defects and randomization of laterality in heart looping. ${ }^{22,23}$ Mutation screening in the studied family showed only a nucleotide change corresponding to a polymorphism described in databases (rs1360294). Small deletions of the KIF3AP coding sequence were also excluded as the cause of the disease in this family. However, inversions, duplications and promoter mutations were not screened for.

Pazour et $a^{24}$ performed a proteomic analysis on flagellar constituents and identified 360 proteins with highly probable flagellar function. Nine of them are localized in 1q23.1-q32.1 and are thus candidate genes for the Kartagener syndrome in family AC-79. In addition, by database searches, they identified 24 human orthologues of proteins associated with Chlamydomonas reinhardtii outer dynein, and 12 genes encoding inner dynein arm subunits. $^{25}$ From these PCD candidate genes, the TXNDC3 is localized in one of the identified candidate loci (7p14.1). 
Screening for other genes with a possible role in leftright patterning is needed to identify the novel gene responsible for Kartagener syndrome. As the intervals are large and no more information can be obtained from this family to reduce the loci, other families with Kartagener syndrome, even if small, would be useful.

\section{Acknowledgements}

We are grateful to all the family members for their valuable participation in this study. These studies were supported by the Interuniversity Attraction Poles initiated by the Belgian Science Policy, network 5/25 and the FNRS (Fonds national de la recherche scientifique) (to MV, a 'Maitre de Recherches du F.N.R.S.'), and the National Foundation For Research in Pediatric Cardiology (to Th.S.). I. Gutierrez-Roelens was supported by a fellowship from FRIA (Fonds pour la formation à la recherche dans l'industrie et dans l'agriculture), and the Patrimoine UCL. The authors thank Anne Van Egeren for her expert technical assistance, and Ms Liliana Niculescu for secretarial help.

\section{References}

1 Kosaki R, Gebbia M, Kosaki K et al: Left-right axis malformations associated with mutations in ACVR2B, the gene for human activin receptor type IIB. Am J Med Genet 1999; 82: 70-76.

2 Bamford RN, Roessler E, Burdine RD et al: Loss-of-function mutations in the EGF-CFC gene CFC1 are associated with human left-right laterality defects. Nat Genet 2000; 26: 365-369.

3 Kosaki K, Bassi MT, Kosaki R et al: Characterization and mutation analysis of human LEFTY A and LEFTY B, homologues of murine genes implicated in left-right axis development. Am J Hum Genet 1999; 64: 712-721.

4 Otto EA, Schermer B, Obara T et al: Mutations in INVS encoding inversin cause nephronophthisis type 2 , linking renal cystic disease to the function of primary cilia and left-right axis determination. Nat Genet 2003; 34: 413-420.

5 Gebbia M, Ferrero GB, Pilia G et al: X-linked situs abnormalities result from mutations in ZIC3. Nat Genet 1997; 17: 305-308.

6 Britz-Cunningham SH, Shah MM, Zuppan CW, Fletcher WH: Mutations of the Connexin43 gap-junction gene in patients with heart malformations and defects of laterality. N Engl J Med 1995; 332: $1323-1329$.

7 Peeters H, Debeer P, Bairoch A et al: PA26 is a candidate gene for heterotaxia in humans: identification of a novel PA26-related gene family in human and mouse. Hum Genet 2003; 112: $573-580$.

8 Robinson SW, Morris CD, Goldmuntz E et al: Missense mutations in CRELD1 are associated with cardiac atrioventricular septal defects. Am J Hum Genet 2003; 72: 1047-1052.

9 Watanabe Y, Benson DW, Yano S, Akagi T, Yoshino M, Murray JC: Two novel frameshift mutations in NKX2.5 result in novel features including visceral inversus and sinus venosus type ASD. J Med Genet 2002; 39: 807-811.

10 Guichard C, Harricane MC, Lafitte JJ et al: Axonemal dynein intermediate-chain gene (DNAI1) mutations result in situs inversus and primary ciliary dyskinesia (Kartagener syndrome). Am J Hum Genet 2001; 68: 1030-1035.

11 Olbrich H, Haffner K, Kispert A et al: Mutations in DNAH5 cause primary ciliary dyskinesia and randomization of left-right asymmetry. Nat Genet 2002; 30: 143-144.

12 Zhang YJ, O'Neal WK, Randell SH et al: Identification of dynein heavy chain 7 as an inner arm component of human cilia that is synthesized but not assembled in a case of primary ciliary dyskinesia. J Biol Chem 2002; 277: 17906-17915.

13 Bartoloni L, Blouin JL, Pan Y et al: Mutations in the DNAH11 (axonemal heavy chain dynein type 11) gene cause one form of situs inversus totalis and most likely primary ciliary dyskinesia. Proc Natl Acad Sci USA 2002; 99: 10282-10286.

14 Broman KW, Weber JL: Long homozygous chromosomal segments in reference families from the centre d'Etude du polymorphisme humain. Am J Hum Genet 1999; 65: 1493-1500.

15 Puffenberger EG, Hu-Lince D, Parod JM et al: Mapping of sudden infant death with dysgenesis of the testes syndrome (SIDDT) by a SNP genome scan and identification of TSPYL loss of function. Proc Natl Acad Sci USA 2004; 101: 11689-11694.

16 Boon LM, Brouillard P, Irrthum A et al: A gene for inherited cutaneous venous anomalies ('glomangiomas') localizes to chromosome 1p21-22. Am J Hum Genet 1999; 65: 125-133.

17 Megarbane A, Salem N, Stephan E et al: X-linked transposition of the great arteries and incomplete penetrance among males with a nonsense mutation in ZIC3. Eur J Hum Genet 2000; 8: 704-708.

18 Ware SM, Peng J, Zhu L et al.: Identification and functional analysis of ZIC3 mutations in heterotaxy and related congenital heart defects. Am J Hum Genet 2004; 74: 93-105.

19 Pennarun G, Escudier E, Chapelin C et al.: Loss-of-function mutations in a human gene related to Chlamydomonas reinhardtii dynein IC78 result in primary ciliary dyskinesia. Am J Hum Genet 1999; 65: 1508-1519.

20 Goldmuntz E, Bamford R, Karkera JD, dela Cruz J, Roessler E, Muenke M: CFC1 mutations in patients with transposition of the great arteries and double-outlet right ventricle. Am J Hum Genet 2002; 70: 776-780.

21 Manning BD, Snyder M: Drivers and passengers wanted! the role of kinesin-associated proteins. Trends Cell Biol 2000; 10: 281-289.

22 Takeda S, Yonekawa Y, Tanaka Y, Okada Y, Nonaka S, Hirokawa N: Left-right asymmetry and kinesin superfamily protein KIF3A: new insights in determination of laterality and mesoderm induction by kif3A $\mathrm{A}^{-1-}$ mice analysis. J Cell Biol 1999; 145: 825836.

23 Nonaka S, Tanaka Y, Okada Y et al: Randomization of left-right asymmetry due to loss of nodal cilia generating leftward flow of extraembryonic fluid in mice lacking KIF3B motor protein. Cell 1998; 95: 829-837.

24 Pazour GJ, Agrin N, Leszyk J, Witman GB: Proteomic analysis of a eukaryotic cilium. J Cell Biol 2005; 170: 103-113.

25 Pazour GJ, Agrin N, Walker BL, Witman GB: Identification of predicted human outer dynein arm genes: candidates for primary ciliary dyskinesia genes. J Med Genet 2006; 43: 62-73. 